\title{
Internet on trains: A multi-criteria analysis of on-board deployment options for on-train cellular connectivity
}

\author{
Bram Naudts, Jonathan Spruytte, Jan Van Ooteghem, Sofie Verbrugge, Didier Colle, Mario Pickavet \\ Dept. of Information Technology (INTEC) \\ Ghent University - iMinds, Ghent, Belgium \\ Email: \{bram.naudts, jonathan.spruytte, jan.vanooteghem, sofie.verbrugge, didier.colle, mario.pickavet\}@intec.ugent.be
}

\begin{abstract}
A train journey could be an ideal moment to answer a phone call, read an e-mail or browse the Internet on a personal device such as a smartphone, tablet or laptop. Unfortunately, a train is a very challenging environment to provide a mobile network service to due to attenuation and bad coverage leading to dropped phone calls and unreachable Internet services. Improving mobile network service on-board trains, however, is costly and should be planned carefully. A multi-criteria comparison of different deployment options is therefore essential toward an optimal investment decision. But, the impact of the choice of on-board distribution system is often overlooked. This article compares three types of on-board signal repeaters side-by-side: IP-based data access points, wideband repeaters and small cells. The differences in terms of network coverage, human exposure, deployment cost, revenue model and telecommunications policy are analyzed for each of them.
\end{abstract}

\section{INTRODUCTION}

We use social media to keep in touch with our friends and relatives. E-mailing, video conferencing, instant messaging, desktop sharing, etc. allow us to communicate and cooperate more efficiently. The proliferation of mobile devices such as laptops, smartphones and tablets allows us to use those services while we are on the move. A train journey is one of those moments that are an ideal time to make use of those services. One does not need to drive, nor does one need to mind the road ahead, the train movement is in many cases quite smooth and one is often comfortably seated. Extending the offer of those services to the train could turn a railway trip into a more pleasant and/or productive experience.

In 2005, Thalys, a European train operating company (TOC) operating high-speed trains between Paris, Brussels, Amsterdam and Cologne started with an offering of onboard broadband Internet. The Beijing-Shanghai High Speed Railway announced in 2011 to provide high-speed Internet for commuters. In 2012, Indian Railways promised to provide Internet in trains. Queensland Rail of Australia rolled out free Wi-Fi to 64 of its trains in 2012 and in Canada, VIA Rail provides a $\mathrm{Wi}-\mathrm{Fi}$ service on-board most of its trains. Worldwide, many other roll-outs have been completed or are being planned.

The offering of an Internet service on trains does however not always meet the quality expectations from the customer. Queensland Rail each customer is allocated a data limit of 20
MB per 4 hour session [1]. On a Thalys train a maximum downlink capacity of $2 \mathrm{Mbit} / \mathrm{s}$ and uplink of $512 \mathrm{Kbit} / \mathrm{s}$ is shared between all customers [2]. Nederlandse Spoorwegen block services such as audio- and video streaming because they consume too much capacity. The solution to provide a better quality-of-service seems simple. Make sure that connections on trains have adequate capacity but the reality is that both technical- and commercial challenges make this problem a hard nut to crack.

Improving coverage is commercially challenging. Public cellular networks are deployed and operated by companies that want to make a profit for their shareholders. Cellular networks (e.g. a $4 \mathrm{G}$ network) are therefore first deployed at areas that provide the best return on investment such as densely populated areas. Next, operators will deploy in strategically important areas such as airports, business parks and shopping centers and last, other populated areas are served by mobile network operators (MNOs) such as small towns, villages and some rural communities. The rail corridor crosses large cities that are covered very well by cellular networks but also remote areas that are not covered because the limited use of wireless connection in those areas does not justify the investment. The provisioning of a train-to-wayside communication link over the public mobile networks is therefore in the first place a commercial problem.

However, commercial challenges are not the only source for coverage gaps. Technical challenges such as the location of rail tracks and attenuation of the mobile signal by train carriages degrade the mobile signal where it is available. Indeed cellular networks are located on places where they can reach populated areas optimally. The nature and location of rail track does limit by itself the opportunity to provide a good service to the rail corridor. The rail track may cross tunnels that block the signal and the ground level of the rail track may be below or above ground level. Covering an area that a train route crosses is therefore not a guarantee for coverage at the rail corridor. On top of that, in areas along the rail corridor where coverage is available, the body of the train carriage worsens the problem. Indeed, a train carriage can be considered to be like a Faraday cage. It blocks radio frequency signals from getting in to (or out of) an area.

Both commercial- and technical challenges are well known 
and have been studied before (section II). To the best knowledge of the authors, the impact of of the on-board distribution system is however overlooked in literature. In this article, we argue that service providers, with the intention to improve service quality to train passengers, should carefully plan their deployment strategy. During the planning process, different architecture options for the on-train distribution link should be taken into account as the choice has a direct impact on both challenges. Therefore, the objective of this article is to provide a multi-criteria comparison of the architecture options for the on-train distribution link. These architecture options are the subject of section III. They have a direct link with the train-to-wayside connection as they can reduce the level of control traffic overhead and hence provide a higher bandwidth to the train passengers (section IV). Human exposure to radio frequency is the subject of section V. Next, we detail the strengths and weaknesses of each architecture in terms of cost of deployment, revenue model and telecommunications policy (section VI and VII). Section VIII summarizes our findings.

\section{RELATED WORK}

Since the proliferation of mobile devices, prominent work on the topic of Internet on trains has addressed several topics. The feasibility of train-to-wayside communication with quality of service over heterogeneous wireless networks was explored in [3]. Although this work is indicative of future developments for reliable train-to-wayside communication, it does not address the challenges that a service operator faces to select the on-board distribution architecture (Wi-Fi or Ethernet is assumed). Noriega et al. [4] present a detailed study on the use of femtocells to enhance the $3 \mathrm{G}$ indoor coverage on trains by setting up femtocells inside the railcars and several wireless interfaces along the roof of the train. The article's results show better signal quality inside the train in terms of delay and packet loss. Unfortunately, the authors do not consider economic factors nor do they consider coverage, telecommunications policy or human exposure. On the other hand, Lannoo et al. [5] study the business model for Broadband Internet on the train. This study reports that the combined usage of different network technologies (i.e. cellular, satellite, WiMAX) is the best solution for offering broadband Internet on the train. The article, which we view as complementary to our work, emphasizes the choice of technology for the train-to-wayside connection while our work focuses on the on-board distribution system. Another technoeconomical paper analyses the economic potential for three different user segments, that is passengers, freight companies and train operator's in-house customers [6]. The authors of the paper recommend to implement the services for different user groups with the same hardware equipment. The work presented in [7] elaborates on this principle of shared resources between services and uses an activity based costing model to calculate the cost per service. The services are provided via IP based data access points and the authors demonstrate that the business case of Internet services on trains can be improved via resource sharing. The different actors that are involved in offering Internet services on trains are analyzed and categorized in three different value network configurations in [8], that is the conventional model, the consortium model and the train operating company model. This last model provides the highest level of openness and flexibility towards service providers. This article takes a different approach to our previous work [7], [8]. The work presented here does not focus on the analysis of the business case for Internet services on trains. It focuses on an analysis of the architecture options for on-board signal distribution and does so by using multicriteria analysis. The analysis includes technical, regulatory and economic criteria.

This article advances the state of the art in the Internet on trains literature by analyzing the different architecture options for an on-board distribution system. We evaluate the different architectures, that is IP based data access points, wideband repeaters and small cells in terms of coverage, human exposure to radio frequency, revenue- and cost model and telecommunications policy.

\section{ARCHITECTURE OPTIONS}

A first architecture option is not installing equipment on the train (base scenario). Passengers connect directly to the wayside and experience, in general, bad quality of service due to signal attenuation and bad coverage. Both the coverage and the attenuation problems must be resolved in order to eliminate or reduce bad coverage. There are two different links to consider. The first link is the link between the wayside and the train-mounted gateway. The second link is the link between train-mounted gateway and the user's mobile device. Both links are interconnected on-board the train by the trainmounted gateway, which is a piece of active equipment with two transceivers. One transceiver is connected to a trainmounted antenna for the train-to-wayside connection and one transceiver is connected to a carriage interior antenna for connection to the handsets. We focus on the evaluation of the on-board distribution link for connection to the handsets.

By installing active equipment on the train, it is possible to reduce the attenuation and improve the coverage. The train is turned into a type of base station, moving alongside the passengers. There are three architecture options for the onboard distribution link:

1) Wideband repeater: A cellular signal repeater is an unmanaged signal amplifier operating in licensed spectrum. For wireless network providers, repeaters are a way of filling coverage gaps and extending reception range. Two flavors of repeaters exist. An analogue repeater does not regenerate data and the repeater is transparent to the surrounding network. They do not allow filtering a signal component from the total received signal. An alternative is a digital repeater which has the ability to filter a signal component from the total received signal and to clear off the noise and interference from the amplified signal.

2) IP data access point: An IP data access point uses unlicensed spectrum. IEEE 802.11, marketed under the WiFi brand name. Wireless access points are the most common 
type of IP data access points.

3) Small cells: Small cells are low-powered radio access nodes that are operator controlled. They typically have a range from ten meters to several hundred meters and are used to eliminate small coverage holes. We focus on femtocells operating in licensed spectrum. A femtocell base station is typically self-configuring and self-managing. The femtocell base stations themselves are connected via a femtocell gateway to the MNO's core network. The gateway comprises a security gateway that terminates encrypted IP data connections from the femtocell. It also comprises a signaling gateway which aggregates and validates the signaling traffic, authenticates each femtocell and interfaces with the mobile network core switches using standard protocols.

The selection of the solution to resolve attenuation losses is tied to the selection of the solution to the coverage problem. As such, in the next section, we consider how the choice of onboard distribution system impacts the coverage of a capacity constrained radio base station.

\section{Coverage}

Passengers do not just want coverage; they want to be able to use broadband services inside the moving train. The quality of service is however constrained by the capacity that a mobile base station can provide. In this section, we argue that the choice of on-board distribution system has an impact on the capacity that is provided to the passenger. The difference is caused by the number of users on-board the train and the amount of control traffic that is generated per active user connected to the operator's mobile base station alongside the rail tracks. To quantify the difference, a GIS based data model was developed in [9].

The geographic information system (GIS) data model contains two main layers. The first layer includes the position of the train tracks which is based on data from the OpenStreetMap project [10]. The second layer includes the position of the cellular antennas which is based on data from the Belgian regulator [11] and the theoretical cell size (see below). When the first layer is overlaid with the second layer the amount of non-covered track becomes apparent.

To estimate the theoretical cell size covered by a base station a link budget has to be determined. The link budget takes all the gains and the losses that occur during the propagation through the medium from the transmitter to the receiver into account. The link budget is needed to calculate the maximum allowable path loss $P L_{\max }$ (in $\mathrm{dB}$ ) to which a transmitted signal can be subjected while still being detectable by a receiver. Once the maximum allowable path loss $P L_{\max }$ is known, the maximum cell size (in meters) covered by a base station can be determined via a path loss model (PLM). A PLM takes into account $P L_{\max }$, the shadowing margin, the frequency, the height of the base station and the height of the mobile base station. Further, when applicable, we apply a Doppler margin of $3 \mathrm{~dB}$ [12] in order to take speeds up to $150 \mathrm{~km} / \mathrm{h}$ into account.

\section{A. Scenario}

The assumptions for the base case of the study draw a scenario where the passengers use the existing 3G network of the Belgian incumbent MNO for Internet access. Two train lines are considered, the InterCity A train line between Ostend and Ghent which mainly passes through densely populated areas and the InterRegio train line between De Panne and Ghent which passes through both densely populated areas and sparsely populated areas. The Erceg $\mathrm{C}$ path loss model [13] is assumed. This PLM corresponds with flat terrain with light tree density and has the minimum path loss. The configuration parameters proposed by the authors of [14] are used to determine $P L_{\max }$ and the cell size. We set the bandwidth requirement according to the peak traffic demand of 2 full crossing trains of 878 passengers each. Based on the adoption of Internet on the trains of Nederlandse Spoorwegen we estimate that around $5.11 \%$ of all passengers on-board the train will be connected to the Internet at the same time [15], [16]. This corresponds to 90 simultaneous users. The market share of the incumbent MNO is $41 \%$ [17] which corresponds to 37 simultaneous users on-board the two trains. For calculation of the cell size the bandwidth requirement is taken into account, the number of active users and the movement of the train. The parameters of Table I are taken into account. Base, repeater, Wi-Fi (1) and femtocell respectively correspond with a scenario in which no repeater, a wideband repeater, Wi-Fi and a femtocell service is deployed. Wi-Fi can be used by all passengers on-board the train (90 users). For the other options only the MNO's subscribers can use the provided service via the MNO's network (37 users). The Wi-Fi service and the femtocell service allow for multiplexing of traffic. As such from the viewpoint of the macrocell, the train-to-wayside connection is a single connection. This is modeled by setting the number of users to 1 during the calculation of the cell size. A wideband repeater cannot multiplex as it repeats the signal at the physical layer. Each connection is therefore a single connection between the MNO's subscriber and the macrocell.

TABLE I

PARAMETERS FOR CONSIDERED ON-BOARD DISTRIBUTION SYSTEM

\begin{tabular}{lllll}
\hline parameter & base & repeater & Wi-Fi & femtocell \\
\hline simultaneous on-board users & 37 & 37 & 90 & 37 \\
train-to-wayside connections & 37 & 37 & 1 & 1 \\
provided bandwidth (shared) & & 6.0 Megabit per second \\
movement of the train & & 100 Kilometer per hour \\
\hline
\end{tabular}

\section{B. Comparison of provided network coverage}

The different on-board distribution systems are compared side by side in terms of bandwidth per passenger and coverage along the train corridor. The bandwidth per passenger reflects the share of bandwidth each passenger can use when the available bandwidth $(6.0 \mathrm{Mbit} / \mathrm{s})$ is distributed equally. The coverage reflects the number of meters of train track on which the minimum amount of bandwidth of $6.0 \mathrm{Mbit} / \mathrm{s}$ as percent of the total track length can be provided. For further clarification, the results are summarized in Figure 1. 


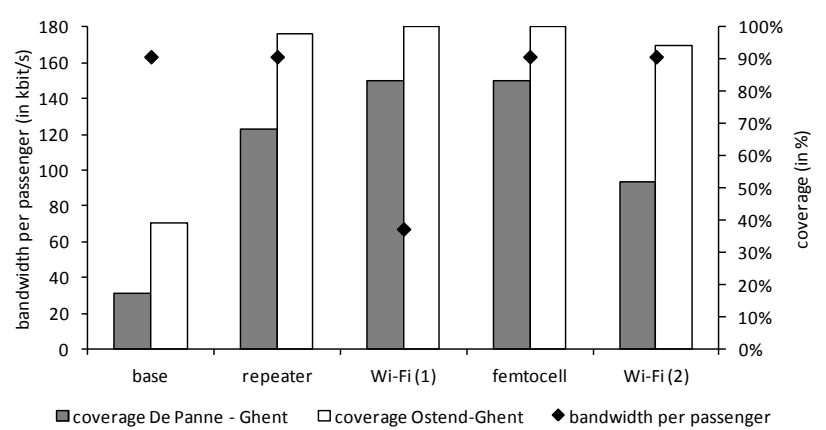

Fig. 1. Coverage rate for the demanded bandwidth (guaranteed) and attainable bandwidth per passenger (equal share) for each of the considered scenarios.

In the base scenario, passengers connect directly to the cellular network and the mobile signal suffers a loss (attenuation by the train carriage) of $18 \mathrm{~dB}$. Coverage is bad for this scenario $(17.17 \%$ and $39.08 \%$ ) due to the high penetration loss.

In the wideband repeater scenario, passengers are still connected directly to the cellular network, however now a wideband repeater is installed. Coverage improves in comparison with the base scenario to $68.38 \%$ and $97.77 \%$, for the train line De Panne - Ghent and Ostend-Ghent respectively. The improvement is completely related to the advantage that signals no longer suffer the $18 \mathrm{~dB}$ train penetration loss.

The Wi-Fi (1) and femtocell scenarios have the highest coverage of all scenarios $(83.22 \%$ and $100.00 \%)$. These options have two advantages. First, the receiving antenna is installed on top of the train which avoids the $18 \mathrm{~dB}$ train penetration loss. Second, all data connections can be aggregated to a single connection (between the train and the cellular network), which results in a lower overhead in the cell and better connections in terms of delay and packet loss.

The attainable bandwidth per passenger is lower for $\mathrm{Wi}-\mathrm{Fi}$ (67.20 kbit/s) than for the other scenarios (163.46 kbit/s). Wi$\mathrm{Fi}$ is available to all passengers, as such more of them will be using the same amount of bandwidth. This corresponds to 90 users in our analyis. For all other scenarios only the MNO's subscribers use the provided bandwidth (only 37 users). For a Wi-Fi service, to provide the same bandwidth to all users a total of $14.71 \mathrm{Mbit} / \mathrm{s}$ has to be provided, this corresponds to a coverage rate of $51.79 \%$ and $94.38 \%$ for respectively the train line De Panne - Ghent and Ostend-Ghent (scenario Wi-Fi (2)).

Femtocell repeaters provide the best coverage and the highest amount of bandwidth per passenger of all architectures.

\section{RADIO FREQUENCY EXPOSURE}

The results of the previous section demonstrate that indoor coverage of a mobile service can be drastically improved by deployment of an on-board repeater. However, the human exposure to radio frequency (RF) electromagnetic fields (EMFs) is from a societal perspective also important. The expansion of the use of wireless networks in everyday live has led to a greater awareness of exposure of the general public to RFEMF and possible adverse health effects. In this section we will assess, based on literature review, the influence of the deployment of a repeater (e.g a wideband repeater, IP data access point or femtocell) on the RF-EMF exposure in a train car.

The authors of [18] characterize the exposure of the general public to RF-EMF due to twelve different radio frequency sources. Twenty-eight different realistic exposure scenarios were characterized and a relevant number of measurements were performed with a personal exposure meter. The scenarios 10 and 11 correspond with measurements on-board the train respectively during the day (from 6am to $6 \mathrm{pm}$ ) and night (from $6 \mathrm{pm}$ to $6 \mathrm{am}$ ) while the train is driving. Measurements were conducted on the IC A train line (Ghent - Brussels) during the day and the train line Brussels - Paris during the night. Of all scenarios, the highest mobile field exposure occurred for mobile scenarios inside a train from uplink signals of GSM (GSM900) and DCS (GSM1800). It was found that the encountered RF-EMF strengths in trains can be much higher than in outdoor or stationary environments. Moreover, for scenario 10, over $99 \%$ of total exposure could be attributed to GSM uplink (UL) and 1\% to GSM downlink (DL) while exposure values for UMTS UL and DL are below the sensitivity of the personal exposure meter. For scenario 11, $94 \%$ of total exposure is attributed to GSM UL, $1 \%$ to GSM DL and almost $1 \%$ to UMTS UL, exposure values for UMTS DL are again below the sensitivity of the personal exposure meter.

Two main factors can be held accountable for this heightened exposure due to uplink radiation: (1) the fast movement of the train, forcing the mobile device to repeatedly connect to a different mobile base station (macrocell) (i.e., a handover); and (2), the metal frame of the train that behaves more or less like a cage of Faraday, significantly attenuating any signal that penetrates it (hence the mobile device has to radiate stronger, in order for the transmitted signal to possess enough power to reach the base station).

The influence on the human exposure to RF-EMF by mobile devices due to the deployment of a Wi-Fi base station is expected to be negligible as approximately $99 \%$ is attributable to GSM UL and DL which is not affected by Wi-Fi. The vicinity of the Wi-Fi base station to the people on the train results in an increased exposure to radiation emanating from the Wi-Fi base station. Depending on the output power of the Wi-Fi base station the total human exposure to RF-EMF could be increased.

A wideband repeater will not reduce handovers but the signal is no longer attenuated due to train penetration. Hence, the mobile device can radiate less strong to reach the base station (uplink). The vicinity of the wideband repeater to the people on the train results in an increase in downlink exposure.

The influence on the human exposure to RF-EMF of the deployment of a small cell in a train is twofold. The vicinity of the cell to the people in the train results in an (on average) substantially increased downlink exposure (i.e., exposure to 
radiation emanating from the base station). However, for the same reason, and also due to the elimination of handovers, the transmit power of the mobile device will be considerably lower, in effect significantly reducing the exposure due to ones mobile device.

Whether the total human RF exposure in the train due to mobile communications is reduced by the deployment of a repeater in the train ultimately depends on several factors, including the output power of the repeater, the number of repeaters in the train, as well as the number of users in the train, and how long they use their devices.

\section{COST AND REVENUES}

In this section the considered on-board distribution systems are compared in terms of cost of deployment and revenue model from the perspective of the cellular network operator. The choice of on-board architecture has a direct link to the investment in the wayside communications equipment. We illustrate this by comparing the number of additional cellular macro cells that have to be deployed per architecture. The installation of active equipment on the train goes hand in hand with the installation of passive equipment such as equipment housing and racks, cabling, inter-carriage links and standardized interfaces. The implementation requires changes to the train and as such requires involvement by the train operating company and the train manufacturer. The installation cost can be very high due to the need for certified technical staff. The installation cost can be reduced when during train construction or train refurbishments space provisions are made for the on-board system.

\section{A. On-board distribution system}

Typically one repeater is installed per train car. For the installation, a roof top antenna will be installed which is connected to the macro cell via a radio link. The on-board repeater is connected to the roof top antenna via the onboard gateway. Distribution of the signal throughout the train carriages is done via a leaky feeder cable which is integrated in the ceiling of the train cars. Typically the roof top antenna and on-board gateway are deployed per consist. A consist is here defined as a set of cars that form a logical unit. One or more consists can be reconfigured to form a train. From a revenue perspective, repeaters are an interesting option for the MNO. First, repeaters are transparent to the network of the MNO. As such subscribers need connectivity to the operator's core network. This allows the MNO to bill the usage of both voice and data services. Second, a digital repeater can selectively transmit frequency bands for amplification of mobile signals within multiple frequency bands allowing MNOs to differentiate their customers from their competitors' customers. This type of differentiation is impossible with an analogue repeater. From a cost perspective it can be noted that digital repeaters are more expensive than analogue repeaters. However, the purchase price of the repeater is only part of the total cost. The installation cost is also considerable as the ceiling of the train car has to be opened to install cabling for connectivity and power supply, to install the roof top antenna, the repeater itself and the leaky feeder cable.

As a rule of thumb for the deployment of Wi-Fi on trains, two wireless access points (WAPs) are installed per train car. The WAPs are connected via a wired network integrated in the ceiling of the carriage to the on-board gateway. The on-board gateway is connected to the roof top antenna via wiring and the roof top antenna is connected to the macro cell via a radio link. From a revenue perspective, standard Wi-Fi does not require connectivity between the user's device and the mobile operator's core network. A MNO can as such not monitor the usage of the service per subscriber or offer additional valueadded services to the user. Customers also consider Wi-Fi as an expected amenity and therefore they have a low willingness to pay for a data service. Wi-Fi does not improve quality of traditional voice calls. This would disfavor the use of Wi$\mathrm{Fi}$ as the on-board access link for voice calls. Eventually the provision of an on-board $3 \mathrm{G}$ or LTE repeater (or pico/ femtocell) might reduce demand for a separate Wi-Fi service. In terms of cost, Wi-Fi access points are cheaper than repeaters and femtocells.

A single femtocell base station can cover an entire carriage. The deployment of a femtocell base station is similar to the deployment of a wireless access point. In contrast to a WAP, a femtocell base station is MNO specific and only the MNO's subscribers can use the service. The subscriber is charged for the services provided via his existing subscription. From a revenue perspective, a MNO can charge for both voiceand data services via the existing subscription. By deploying femtocells, the MNO can also differentiate his offering from that of other MNOs which could possibly lead to a higher retention rate of existing customers and new customers joining because they appreciate the quality provided. The deployment cost of a femtocell service on-board a train car is similar to the cost of providing a Wi-Fi service. To cover all passengers, a femtocell base station has to be deployed for each mobile network operator or MNOs should allow a type of roaming service on their network. To offer a femtocell service, an operator is also required to operate a femtocell gateway to which the femtocell base station can connect. As a femto gateway is a highly specialized, expensive device that can support hundreds of thousands of femtocell base stations, the deployment of a femtocell gateway cannot be justified in economic terms when this device has to be purchased for an on-train femtocell service alone. Femtocells should therefore fit within the existing strategy set out by a mobile network operator.

\section{B. Cellular train-to-wayside communication}

Mobile network operators have a legacy network of macro cells to service their subscribers from. Raising the base station density along the rail corridor is an expensive option for the operator. The authors of [19] estimate that site acquisition and construction has a Capital Expenditure (CapEx) cost of $75.000 €$. The CapEx of a cellular base station is estimated at $33.000 €$. Further, the annual Operational Expenditure (OpEx) 


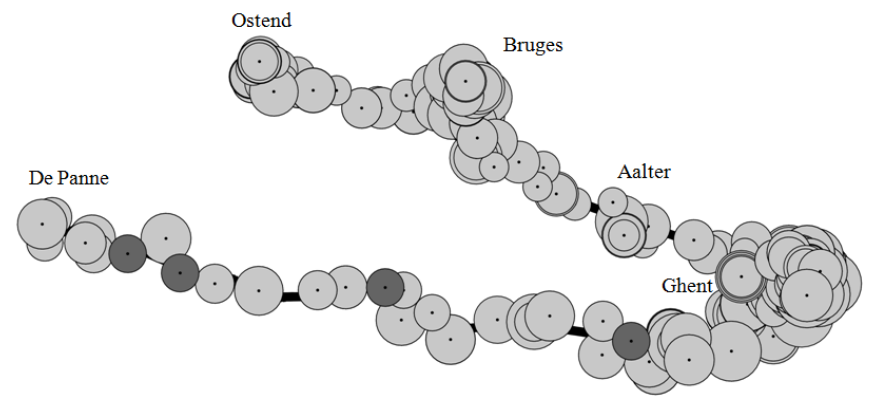

Fig. 2. Wi-Fi (1)- and femtocell scenario: The train lines Ostend - Ghent and De Panne - Ghent with the location and cell size of all antennas that cross the train line. On the train line Ostend - Ghent coverage is in general excellent apart from a coverage gap before and after Aalter. On the train line De Panne - Ghent coverage is worse with more coverage gaps. To reach $95 \%$ coverage 4 additional antennas have to be deployed which are depicted in dark gray.

is estimated at $17.5 \%$ of CapEx. The mobile backhauling cost is another substantial cost category which is even higher in remote areas. The deployment of macro cells in remote areas is clearly an expensive option which does not fit with the operator's need of a cost-effective network. Hence, the number of extra macro cells should be minimized. Section IV illustrates that the choice of on-board architecture does affect the provided coverage. To improve coverage further on the IC A line between Ostend and Ghent and the IR line between De Panne and Ghent a mobile network operator could improve his existing network. We assume that the MNO wants to improve coverage rate for the demanded bandwidth up to $95 \%$ and used a genetic algorithm described in [9] to calculate for each of the three types of architectures how many extra macro cells are required to reach a coverage rate of $95 \%$.

The initial coverage for the base scenario is $39.08 \%$ on the train line Ostend-Ghent and $17.17 \%$ on the train line De Panne - Ghent. To reach 95\% coverage on the train line OstendGhent, 37 additional radio base stations have to be deployed. On the train line De Panne - Ghent 68 additional antennas are required. This high amount is decimated when the losses due to train penetration are reduced via the installation of a repeater. When a wideband repeater is deployded, 7 additional antennas are required. For the Wi-Fi (1)- and femtocell scenario, 4 additional antennas are required. These results are naturally specific for the train lines under consideration here but the general trend is a guideline to other train lines. For further clarification, Figure 2 illustrates one of the considered scenarios.

\section{TeleCOMMUNiCATIONS POLICY}

Telecom regulators are in many cases the entity responsible for the licensing of the electromagnetic spectrum to the MNOs. The licenses can include obligations for coverage of an area or a share of the population. In this section, we compare the on-board distribution systems in terms of how they are being affected by the applicable regulation.

Wi-Fi uses the unlicensed 2.4GHz-band (IEEE $802.11 \mathrm{~g}$ and IEEE $802.11 \mathrm{n}$ ) or the 5Ghz-band (IEEE 802.11n). The deployment of an on-board Wi-Fi distribution system does not require involvement of a mobile network operator. A drawback of unlicensed spectrum is that it is not free from interference. However, the train carriage is a good signal attenuator reducing interference from transceivers outside the train to a minimum.

Wideband- and femtocell repeaters operate in licensed spectrum leased by an MNO. Installation or use of a wideband repeater device is unlawful unless the equipment is compliant with all relevant regulatory requirements. In practice, only the MNOs are licensed to use a repeater that transmits in the cellular frequency bands. Femtocell devices may be installed in user premises. A key feature is that they are monitored and controlled by the host network to ensure operation within the terms and conditions of the network operators' licenses under which they are authorized. Other types of stand-alone repeaters which are not covered by the network license nor exempted from licensing are not authorized. Hence, the use of wideband- and femtocell repeaters on trains will necessarily involve working in collaboration with MNOs. The regulator can however review their regulatory position and exempt the use of licensing for mobile repeaters specifically for application in trains.

Spectrum licenses are only valid within the national border while many train cars may from time to time cross national borders. Wideband- and femtocell repeaters should therefore stop operating when a border is crossed unless there is an agreement with the MNO of the other country.

Wideband- and femtocell repeaters have the advantage of improving coverage for both voice and data but they require the involvement of MNOs. Currently there is no incentive for an MNO to extend coverage to the train as the potential revenue increment cannot justify the extra expenditure. This lack of incentive applies for data traffic as well as voice. A controversial option could be that the regulator adds an obligation to cover the rail corridor in future licenses.

\section{CONCLUSION}

Improving mobile service on trains is costly and should be planned carefully. The impact of the on-board system was until now overlooked. A multi-criteria comparison has been conducted to the deployment of different on-board distribution architectures. The results of this analysis are summarized in Table II. For most criteria, that is coverage, cost, revenue and human exposure, a femtocell service has an advantage to or at least performs equal to the other architecture options (wideband repeaters and IP data access points). A Wi-Fi service however does not require the involvement of an MNO as the service uses unlicensed spectrum. This may explain the choice for this architecture by most Internet on train deployments. Femtocells, in contrast, operate just like wideband repeaters in licensed spectrum. Hence they require the involvement of the MNO. Unfortunately, MNOs lack an economic motive to extend coverage to the train. We therefore argue to strive for closer collaboration between the mobile network operator and the train service operator and a regulatory environment that spurs the deployment of Internet on trains. 
TABLE II

SIDE BY SIDE, MULTI-CRITERIA COMPARISON OF ON-BOARD DISTRIBUTION ARCHITECTURES FOR ON-TRAIN CELLULAR CONNECTIVITY

\begin{tabular}{llll}
\hline Criteria & Wideband repeater & IP data access point & Femtocell \\
\hline voice calls (GSM) &,+ reduction in attenuation loss & $=$, direct connection to the wayside & $\begin{array}{l}+++, \text { reduction in attenuation loss } \\
\text { and multiplexing of voice calls }\end{array}$ \\
data traffic &,+ reduction in attenuation loss & $\begin{array}{l}++, \text { reduction in attenuation loss } \\
\text { and multiplexing of data traffic }\end{array}$ & $\begin{array}{l}+++, \text { reduction in attenuation loss } \\
\text { and multiplexing of passenger traf- } \\
\text { fic }\end{array}$
\end{tabular}

radio frequency downlink exposure radio frequency uplink exposure

cost of on-board equipment

revenue model

telecommunications policy

-, vicinity of base station to the people on the train can substantially increase downlink exposure

+ lower transmit power: mobile devices radiate less strong to reach $=$ negligible, as it has no effect on GSM uplink

++ lower transmit power: mobile devices radiate less strong to reach the mobile base station, elimination of handovers

The equipment cost is a small part of the total cost. If the implementation requires changes to the train the installation cost can be very high due to the need for certified technical staff. The cost for extra wayside macro cells is a magnitude higher and should be minimized by the choic of on-board equipment.

$=$, a digital repeater is more expensive than an analogue repeater, cost of the repeater depends on the number of frequency bands supported, typically one repeater per consist and leaky feeder for signal distribution through train cars

,+ charged for via mobile subscription, both voice and data services possible as well as value-addes services

-, operates in licensed spectrum, does require involvement from the mobile network operator
$=$, comparable in terms of cost with femtocell access point and does not need expensive core equipment, typically two WAPs per train car, cost can be shared between MNOs

-, hard to charge for directly, only data service possible but passengers are not willing to pay for $\mathrm{Wi}-\mathrm{Fi}$, not possible to offer valueadded services

+ , operates in unlicensed spectrum, does not require involvement from the mobile network operator
$=$, comparable in terms of cost with IP data access points but requires one per MNO and expensive core equipment, typically one per train car

++ charged for via mobile subscription, both voice and data services possible as well as value added services, best quality-ofservice

-, operates in licensed spectrum, does require involvement from the mobile network operator

\section{ACKNOWLEDGMENT}

This research was carried out as part of the iMinds RAILS project. This project is co-funded by iMinds a research institute founded by the Flemish Government. Companies and organizations involved in the project are Televic Rail, Uify, Option and Belgacom, with project support of IWT.

\section{REFERENCES}

[1] QueenslandRail Wi-Fi terms and conditions of use. [Online]. Available: http://www.queenslandrail.com.au/RailServices/City/Pages/WiFitermsofuse.aspx

[2] 21Net Thalys. [Online]. Available: http://www.21net.com/\#!thalys/cd4p

[3] D. Pareit, E. Van de Velde, D. Naudts, J. Bergs, J. Keymeulen, I. De Baere, W. Van Brussel, C. Vangeneugden, P. Hauspie, G. De Vos et al., "A novel network architecture for train-to-wayside communication with quality of service over heterogeneous wireless networks," EURASIP Journal on Wireless Communications and Networking, vol. 2012, no. 1, pp. 1-30, 2012.

[4] P. Noriega-Vivas, C. Campo, C. Garcia-Rubio, and A. RodriguezCarrion, "MOFETA: a network architecture based on MObile FEmtocells to enhance cellular connectivity on TrAins," in Communication Technologies for Vehicles. Springer, 2012, pp. 174-185.

[5] B. Lannoo, J. Van Ooteghem, D. Pareit, T. Van Leeuwen, D. Colle, I. Moerman, and P. Demeester, "Business model for broadband internet on the train," Journal of The Institute of Telecommunications Professionals, vol. 1, no. 1, pp. 19-27, 2007.

[6] V. Riihimaki, T. Vaaramaki, J. Vartiainen, and T. Korhonen, "Technoeconomical inspection of high-speed internet connection for trains," Intelligent Transport Systems, IET, vol. 2, no. 1, pp. 27-37, March 2008.

[7] B. Naudts, J. Van Ooteghem, S. Verbrugge, D. Colle, and M. Pickavet, "Insights in the cost of continuous broadband internet on trains for multi-service deployments by multiple actors with resource sharing," EURASIP Journal on Wireless Communications and Networking, vol. 2014, no. 1, pp. 1-18, 2014.
[8] B. Naudts, J. Van Ooteghem, B. Lannoo, S. Verbrugge, D. Colle, and M. Pickavet, "On the right tracks? Continuous broadband internet on trains," Journal of the Institute of Telecommunications Professionals, vol. 7, pp. 31-36, 2013.

[9] J. Spruytte, B. Naudts, J. Van Ooteghem, S. Verbrugge, D. Colle, and M. Pickavet, "Planning omni-present networks of the future: Covering the smart city with an ubiquitus network fabric," unpublished.

[10] OpenStreetMap. [Online]. Available: http://www.openstreetmap.org/

[11] Belgisch Instituut voor Postdiensten en Telecommunicatie. [Online]. Available: http://bipt.be/

[12] L. Verstrepen, W. Joseph, E. Tanghe, J. Van Ooteghem, B. Lannoo, M. Pickavet, L. Martens, and P. Demeester, "Making a well-founded choice of the wireless technology for train-to-wayside data services," in Telecommunication, Media and Internet Techno-Economics, 9th Conference, Proceedings, June 2010, p. 7.

[13] V. Erceg, L. J. Greenstein, S. Y. Tjandra, S. R. Parkoff, A. Gupta, B. Kulic, A. A. Julius, and R. Bianchi, "An empirically based path loss model for wireless channels in suburban environments," Selected Areas in Communications, IEEE Journal on, vol. 17, no. 7, pp. 1205-1211, 1999.

[14] M. Deruyck, E. Tanghe, W. Joseph, and L. Martens, "Modelling and optimization of power consumption in wireless access networks," Computer Communications, vol. 34, no. 17, pp. 2036-2046, 2011.

[15] "Jaarveslag 2010," annual report, Nederlandse Spoorwegen.

[16] "Gratis internet in de trein krijgt vervolg," press release, Nederlandse Spoorwegen, apr 2013

[17] "Annual report 2013," annual report, Belgacom.

[18] W. Joseph, G. Vermeeren, L. Verloock, M. M. Heredia, and L. Martens, "Characterization of personal rf electromagnetic field exposure and actual absorption for the general public," Health physics, vol. 95, no. 3 , pp. 317-330, 2008.

[19] Z. Frias and J. Pérez, "Techno-economic analysis of femtocell deployment in long-term evolution networks," EURASIP Journal on Wireless Communications and Networking, vol. 2012, no. 1, pp. 1-15, 2012. 